\title{
Asset trading under non-classical ambiguity and heterogeneous beliefs
}

\author{
Polina Khrennikova*, Sudip Patra ${ }^{\dagger}$
}

January 15, 2019

\begin{abstract}
We propose discrete time asset trading framework based on quantum probability formalism that represents well the ambiguity of agents in respect to the fundamental values and price states of the traded assets. Divergence of beliefs alike classical finance frameworks (e.g. works by Harrison and Kreps, 1978 [24]; Scheinkman and Xiong, 2003 [50]) produces different expectations of agents about the future price distribution of the traded risky asset. The model accounts for the emergence of heterogeneous beliefs from agents' ambiguity about both the future asset price states and the fundamentals, as opposed to the strands that attribute heterogeneous beliefs to asymmetric information and different, yet firm prior beliefs about stochastic processes over fundamentals. The introduced quantum probability paradigm allows to depict a genuine ambiguity of agents in respect to the future realization of payoff relevant variables and prices. There are two sources of ambiguity: i) the imperfect market knowledge of agents, manifest in a divergence of ambiguous priors, ii) uncertainty about the probability distribution of price states and dividends in the next trading period. Agents update their beliefs via Born rule (instead of Bayesian update) when observing the realised price outcomes and dividend signals. An important feature relates to individual traders' not possessing a joint probability distribution over the payoff relevant variables and price outcomes that brings up attraction, respective aversion to ambiguity in their interpretation of public signals. On the level of the composite model of stock exchange, formed by the expectations of two ensembles of agents, an interference term can serve as a quantitative testable
\end{abstract}


prediction in respect to the excess volatility of asset prices created by traders' optimistic and pessimistic beliefs.

keywords: asset trading; speculative asset prices; heterogeneous beliefs; ambiguity; state dependence; quantum probability.

\section{Introduction}

There is a long standing debate in the standard neoclassical finance literature on the impact of uncertainty on financial asset prices. The best known models going back to Miller, [44] have held that uncertainty is represented by some measure of divergence of investors' opinions, grounded in the presence of information asymmetries in incomplete markets. Miller conceived that "the very concept of uncertainty implies that reasonable men differ in their forecasts", [44], p.1151. Such divergence of states of beliefs causes the deviation of asset prices from the so called fundamental value as predicted by the asset pricing models such as CAPM. ${ }^{1}$ Since the central premise of rational expectations (RE) models is homogeneity of investors' beliefs, along with some restricted perfect market conditions, arbitrage is not possible in the long run and asset prices do not deviate from what the fundamentals predict. This is also an important implication of the seminal EMH (Efficient Market Hypothesis) by [23] that builds upon the notion of common knowledge of market actors about all the available information as well as singularity in mapping from the observed fundamentals to asset prices. As a consequence, in the absence of new information all agents agree on the fair price of a risky asset. When new information about payoff relevant variables arrives, the agents react to the price relevant news following a classical Bayesian update scheme. ${ }^{2}$ The assumption of equal priors and homogeneous posterior beliefs, as a result of common knowledge that is central to the above finance frameworks, was shown to be a rather idealized postulation, in particular, when one deals with such a complex system as finance market containing multiple sources of noisy information, as opposed to an observation of some simple events, such as a coin toss. For decades finance literature was preoccupied with the identification of the sources of heterogeneous beliefs and their impact upon asset prices, associated with periods of high volatility and

\footnotetext{
${ }^{1}$ Capital asset pricing model establishes the 'right' rate of return for holding a risky asset that together with expected cash flow projections, or all expected future dividends allows to assess a current 'fair' price of that asset.

${ }^{2}$ Under asymmetric information with non-biased private signals, rational agents would ideally be able to infer other traders' information from the observed asset prices and hence a fully revealing rational expectations equilibrium is attained, [21].
} 


\footnotetext{
${ }^{3}$ Risk attitudes do not affect risk premium and the impossibility of short sales does not allow pessimists to short the asset, thereby giving rise to bubbly and, in other trading periods, as belief states switch, to deflationary pressures.
} 
formation processing and belief formation under informational ambiguity of agents about the dividend signals and prices in the next trading period. It is important to note that agents form separate beliefs about the price evolution and dividend signal distribution, contrary to the assumptions of classical DDM (Dividend Discount Models) in which only the future dividend streams matter for the current asset price. The state space of the agents, the so called Hilbert space, consists of the subspaces related to the outcomes of price and dividend observables, which act upon the belief state vector $(\psi)$ of each agent. QP calculus relaxes the assumption of a joint probability distribution over prices and fundamentals that is axiomtized in classical probability theory via the commutativity rule of probability distributions. ${ }^{4}$ Agents can be affected by non-commuting observables (given by random variables in classical probability theory), associated with dividends and prices. A quantum formula of total probability introduced in eq. (2) contains an additional interference term that mathematically depicts the interference effects in agents' beliefs. Positive interference of probability amplitudes pertains to overweighting of probability of price value. Negative interference gives raise to pessimistic beliefs, manifest in under-weighting of the probabilistic prognosis in respect to the realizations of future increase in price and dividends. Non-classical ambiguous beliefs produce trading preferences associated with upwards volatility in respect to the fundamental valuation based on RE. As beliefs of agents oscillate between the trading periods, a state transition to pessimistic beliefs creates trading preferences that contribute to deflationary pressures.

Given the importance of the non-singularity of beliefs in affecting the asset price volatility we operate with two ensembles of agents. Their heterogeneous ambiguous beliefs are caused by optimism or pessimism affect. ${ }^{5}$ We also conceive that the cognitive states of pessimism and optimism trigger ambiguity attraction respective aversion among agents, when evaluating the asset price distributions in the next trading period, which is in line with findings in [49]. In the absence of dividend signals, agents' ambiguity attraction in creates beliefs that the asset price will go up in the coming trading period. Upward price volatility emerges and the risky asset price raises above its fundamental value. The coefficients of interference allow to quantify the degree of overvaluation respective undervaluation by the agents. Divergence of

\footnotetext{
${ }^{4}$ Non-commutativity brings up a non-satisfaction of the core rule of classical probability theory, the formula of total probability, [39]. A deviation from classicality in information processing, given by a violation of the independence axiom of SEUT has been detected in a large body of studies in economics, starting with the seminal Ellsberg paradox.

${ }^{5}$ In the proposed model uncertainty about divided distribution is assumed to be symmetrical, and the heterogeneity in the limiting probability distribution is solely due to divergence in the prior ambiguous beliefs.
} 


\footnotetext{
${ }^{6}$ In Appendix 8 we espouse a multi-period belief evolution setup, where the internal belief evolution can create differences in information update, following the observation of a sequence of dividend and price outcomes.

${ }^{7}$ We focus here on quantum dynamical models. A survey of the achievements of models borrowed from physics in the fields of economics and finance can be found in the monograph by [41] as well as recent works by [31] and [3]. The latter work examines the potential of physical frameworks to serve as alternative financial models to model asset price formation beyond EMH.
} 
To sum up, our setup is aiming to enrich the field of above reviewed contributions of QP models to capture asset pricing under two deviations from RE given by, i) non-classical information processing and heterogeneity in opinions, ii) informationally incomplete markets. To the best of our knowledge, there are no contributions to speculative asset pricing that focus on these two phenomena by representing agents' opinion update by QP probabilistic measures. The paper is organized as follows: in the next Section 2 we sketch the mathematical differences between the classical and quantum probability information processing schemes. We motivate the usage of quantum probability framework as a descriptive DM model for agents' decision making under uncertainty. In Section 3 we provide a mathematical framework underpinning the all-important distinction of quantum versus classical modeling of heterogeneous beliefs under uncertainty. In Section 4 we illustrate the geometric properties of agents belief state evolution, and introduce belief and price behaviour operators. In Section5 we define the Born rule of information update in the QP measurement scheme. In Section 6 we summarize the possible empirical predictions of our framework, and in Section 7 we conclude.

\subsection{Related Literature}

It has been shown in a large body of finance studies that heterogeneous and irrational beliefs can generate speculative pressures on capital markets manifest in asset price bubbles, which may sustain for long periods, see accounts in [51]-citeShiller2. A survey of bubble emergence emphasising the role of divergent beliefs on agents' speculative behaviour can be found in [56]. The impact of heterogeneous beliefs is not the only cause of the existence of inflationarydeflationary pressures on asset prices. Excess volatility can also take place under rational expectations, caused for instance by dynamic inconsistency of agents and credit constraints, [43]. Shiller documents:"speculative bubble (is) a natural consequence of the principles of social psychology coupled with imperfect news media and information channels." [52], p.1487.

The 'agree to disagree' phenomenon and its effects on asset trading, whereby speculative behaviour can emerge was firstly formalized in the asset trading framework by [24], followed by a dynamical representation in [50]. The existence of disperse beliefs is reflected in deviations from the RE equilibrium price that is based on homogenous expectations of agents. ${ }^{8}$ In partially

\footnotetext{
${ }^{8}$ Seminal 'no-trade theorem' due to [53] postulates the impossibility of the emergence of bubbles under RE. Under the existence of symmetric information and classical information processing scheme, traders are aware of the true probability distribution of future returns and hence, the fundamental value of assets is a common knowledge. Any time any bubbly
} 
revealing equilibriums of asset trading, the agents can be aware of other agents' possessing divergent beliefs, due to different private information sets, or due to overestimation of the informativeness of some public signals. This awareness can trigger trading behaviour directed to benefit from perceived overvaluation of assets by other less rational agents, see asset trading under 'beauty contest' by [11]. A similar assumtion about the lack of rationality of other agents is made in [50]. Among other, the models by [24], [50], [11], assume symmetric upcoming information, yet the agents can make different forecasts due to optimism and different priors. In particular the work by [11] addresses higher order beliefs (i.e. the beliefs of investors about the beliefs of others) under symmetric information and different priors, expanding on the seminal work of Harsanyi.

Optimism as a cognitive feature of the decision makers also contributes to a divergence in prior belief states (i.e. the degree of optimism will cause different, yet firm prior beliefs among ensembles of agents under symmetric information) as postulated in [24]. The heterogeneity in beliefs is an important trigger of speculative trade, as beliefs about asset valuation switch between agents over the trading periods. Frameworks by [45] and [11] also formalize asset trading with divergent prior opinions. Learning among agents can occurs over time, as agents observe a sequence of dividends and prices converge to the fundamentals over long term, [1], [19], [45]. At the same time, agents can update separately their price expectations and dividend expectations, based on the observed market outcomes, [1]. Finally, managerial decision making under different opinions is exlpored in [19]. In their work the role of asymmetric information and optimism, characterising the divergence of beliefs in principal-agent relationships can create under-investment. The stream of literature that explores emergence of adverse selection due to asymmetric information is also broad based with a focus on identifying the degree of adverse selection impact on the finance market performance. ${ }^{9}$

Divergence of opinions can be coupled with adverse selection, where some traders lack the private information possesed by other traders. A widely used measure of the existence of adverse selection is the bid-ask spread, that quantifies agents' attempts to minimise their possible losses, due to the lack of complete information, [20]. Contribution by [9] addresses the impact of adverse selection by devising a continuous dynamical asset pricing model for

trends are due to emerge, an agent can infer information from prices by possesing the same likelihood function as other agents.

${ }^{9}$ Adverse selection problem would naturally not exist in efficient markets, since less informed investors would follow the more informed ones, since the rationality of all agents is common knowledge, [21]. 
rational investors under incomplete, but symmetrical information. ${ }^{10}$ In addition to the above reviewed causes, heterogeneous beliefs can also emerge as a result of non Bayesian information processing. As mentioned, the divergence in beliefs can result from different 'biases' that cause the non-classical processing of information where 'noise' is present often categorised as optimism and pessimism. The 'noise' in the estimation of the dividend rate can be captured via a Markov process following [38], with a coefficient $\pi$ that serves to create optimism $(\pi>0)$, or pessimism $(\pi<0)$ in respect to the variance of the drift factor $\mu$. Another cause behind the violation of Bayesian update and other axioms of classical probability can be due to agents' employing a fundamentally different mechanism of information updating under uncertainty, rather than information processing rationality implied by the neo-classical normative decision theories. In real finance setting, the agents often cannot reach resolution from uncertainty about the realization of future states of the world. The agents can trade, while being ambiguous about the future probabilistic distribution of asset price returns, or have ambiguous expectations about the informativeness of private signals. Belief formation and update under ambiguity and ambiguous information is already well researched in asset trading theories, mainly via the usage of 'max-min expected utility' (MEU) and a dynamical modification thereof, see [13], [16], and references herein. Agents can exhibit ambiguity aversion and ambiguity attraction that affect their preference formations as espoused in $([18],[36],[17])$. In recent contributions, [14] also seek to examine the effect of an interaction of public and private information upon asset prices via an introduction of two ensembles of agents. The informed agents exhibit ambiguity aversion and hence bias the full revelation of prices for the other (less informed) ensemble of agents. Further, the effect of short sale constraint upon return volatility is formalized by [46], showing that as the private information becomes more ambiguous, negative effects on asset price dynamics emerge when coupled with short sale bans. Finally, work by [30] extends the analysis of bubbles to include overtime regime shifts in the fundamentals to provide the necessary conditions for bubble emergence in derivative markets. To round up, classical probabilistic heterogeneous beliefs in the sense of [39] are likely to be attributed to: i) divergence of prior beliefs due to some cognitive differences of agents; ii) lack of common knowledge caused by asymmetric, or uncertain information, and iii) non classical mode of information processing, such that Bayesian reasoning is not employed due to some 'biases' or 'noise'. The last case demands a

\footnotetext{
${ }^{10}$ In this context, it is important to observe how the effect of uncertainty on prices differs from the standard risk attitude divergence. Different preferences about the required risk premium can yield divergence in required discount rate among investors and hence, their affect their valuation of the fair price as shown in the early work by [42].
} 
relaxation of some of the classical probabilistic axioms, such as distributivity and commutativity. Under uncertainty and information asymmetry, the beliefs of agents can be also ambiguous and contextual factors can give raise to ambiguity aversion that affect their preference formation, [13], [17], [18], [36]. Ambiguity attraction is less researched in ambiguity based asset trading works, yet can be closely related to optimistic behaviour under uncertainty, [2], [49]. We note that the reviewed frameworks also make opposite predictions in respect to the emergence of speculative trading; while ambiguity aversion implies under-pricing, heterogeneous beliefs and optimism can lead to overpricing, thus creating bubbles. The present framework aims to unify these predictions, by relating instances of overpricing and under-pricing, via the transformation of agents' heterogeneous (beliefs) in different time periods that follow the rules of projective measurements of quantum probability.

Ambiguity impact on decision preferences, as well as information processing under ambiguity has been well addressed in recent studies in economics and decision theory via the usage of QP, rather than classical theory of probability and stochastic processes, for instance, [47], [12], [26], [33] address the emergence beliefs and preferences under non-classical ambiguity that describe well the violation of classical Bayesian updating scheme in 'Savage Sure Thing principle' problems and the 'agree to disagree' paradox. In [27] additional empirical evidence on non-consequential preferences in investment choices is collected and accommodated in QP framework. A QP model for order effects is formalized in [55] that accounts for state dependence in information processing. Ellsberg and Machina paradox behaviour from ambiguous beliefs is formalised in [2] with aid of QP calculus. The work by [35] proposes decision making scheme via the usage of creation and annihilation operators from the quantum information theory. The existence of 'zero prior paradox' that challenges Bayesian updating from uninformative priors is attested and solved with the aid of projective scheme of information update in work by [10]. Finally, [15] apply the QP formalism of information update under the study of persuasion in investment and consumption choice, showing that the non- satisfaction of the recursive dynamic consistency of choices can be mathematically depicted through incompatible information observables. The usage of QP as an alternative descriptive (and potentially normative) framework of preference formation under uncertainty can be justified given the body of empirical evidence in the above mentioned and related studies in economics and psychology. The findings affirm an existence of different attitudes among decision makers towards ambiguity and risk that are not in accord with the SEUT or non-linear probabilistic transformations thereof. The main advantage of QP is that it is a complete probabilistic framework that allows to accurately depict indeterminacy of agents and its overtime 


\footnotetext{
${ }^{11}$ For an extended mathematical analysis cf. monographs by [12], [26] and survey by [48].
}

dynamics. The axiomatic of quantum probability is based on a different mathematics, where QP is by definition a non set-theoretic probability theory relaxing the distributivity axiom (exclusivity of events and their additivity) and commutativity (context independent joint probability distribution). The prior ambiguous beliefs are modeled in QP framework as superposition of agents' belief states. Superposition state allows to reproduce the ambiguity of agents associated with the probability distributions of future asset prices and dividends. The initial belief state is an indefinite, 'superposition' state of various probability distributions, or preferences and interference effects can be present. We would like to emphasize that superposition representation is fundamental to differentiate between the classical and quantum belief state description. Our proposal, along with the existing studies using QP based decision theory, is that the superposition representation of probability amplitudes captures better the ambiguous beliefs than a classical ensemble description. The quantum probability is obtained from quantum wave function (probability amplitudes) that can also vacillate over time. ${ }^{11}$ The random variables in QP are given by observables and the events by subspaces of a Hilbert space, rather than by the sigma algebra on the probability sample space. To round up, the QP approach to information processing is considered in the literature as a viable mathematical framework of information processing that can also be applied to agents' information processing on the financial market. In proposed model we aim to develop the belief formation scheme about fundamentals and price in a discrete time setup and describe the emergent non-classical asset price equilibrium today. In the next sections we continue with the formulation of the quantum probabilistic setup, to devise a model of belief state formation and update under uncertainty in the context of asset trading.

\section{Model of trading under uncertainty in quan- tum probability (QP) framework}

The aim of the framework is to elucidate how non-classical heterogeneous beliefs of market participants create interference effects that amplify the trading optimism and in other periods trading pessimism. The state of the market participants changes after an informational context and the interference affects can create amplification of buying respective selling, even under no upcoming information,[51]-[52]. The states of participants update (e.g. af- 
ter observation of previous trading dynamics) and trading bids and asks can create sudden price moves for no apparent reason.

In our model we capture the amplification mechanism via non-classical belief indeterminacy, where the ambiguous beliefs about the price states can interfere with the ambiguous beliefs on fundamentals' distribution.

Before we present information processing under QP under asset trading, we review in a simple dichotomous scheme the information update under uncertainty that lies at the heart of SEUT.

\subsection{Agents' decision making: classical probability based information processing}

In the classical probabilistic information update, each infinitely lived agent from the population $N$ is endowed with an initial pure belief state with respect to one risky asset value $I$ in question. Every agent operates in belief space for dividends, $D$ and a belief space, $K$ for asset price realizations, upon which she makes the hold and sell decisions. The composite state space that includes all possible asset price and dividend realisations up to the decision time $t$ is $\Omega^{t}$, where $t=0,1,2 \ldots$ are some discrete points in time. Hence, every agent is a composite system of $D$ and $K .^{12}$ To simplify the exposition of probabilistic update of the model at this stage we consider two types of beliefs about the discrete asset price movements, $P_{+}$and $P_{-}$, that correspond to agents' decisions to buy or sell the asset now. There is a dividend signal that we denote in dichotomous form as positive or negative $\left(S_{+}\right.$or $\left.S_{-}\right)$related to the asset valuation. The arrival of such as signal in classical finance theory changes the initial belief state of the agents in a Bayesian updating scheme. What is important, in classical probabilistic framework the random variables corresponding to the asset prices, and informational signals are partitions of the same sample space, i.e. the agents have a joint probability distribution of signals and corresponding price outcomes, due to Kolmogorov [39] probability theory. The agents can also form a joint probability distribution of all asset price states given the dividend realisations in a given decision making context.

The key rules of classical information processing scheme (due to SEUT and its modifications based on classical probability theory) imply that the agents make a joint probabilistic evaluation of all possible signal-events and the corresponding consequences. These probabilities are corresponding to

\footnotetext{
${ }^{12}$ On the finance market only the actions and decisions of actors are visible through the changes in asset prices. Hence, the beliefs about asset prices are given by the expectations of the agents about the asset price given the future dividends. Each realization of the dividend at time $t=1$ allows to assume as specific value of the asset price.
} 
firm beliefs and no indeterminacy in agent's expectations is present at each time point. If the probability of the price increase is higher than of a price decrease, the risk neutral decision maker will have a higher expectation value from holding the asset between $t=0$ to $t=1$. For now, we assume that the price increase and decrease size in next trading period is of the same magnitude. We also assume that in the pricing of the asset the agent would use some risk neutral discount factor $r$, i.e. $P_{t=0}=e^{-r \Delta_{t}} E_{t=1}\left(P_{t=1}\right)$. The expectation value for price at $t_{1}$ is obtained by agents via analysing the probabilistic distribution of fundamentals (approximated by dividends). After the dividend outcome news are observed, each agent is able to evaluate in a Bayesian mode the conditional price state distributions. We remark that $S \pm$ denotes the news about the dividend increase or decrease in the next trading period. We note that alike the classical finance frameworks the actual dividend distribution causes a proportional decrease in the price value on the ex-dividend date. In incomplete markets some signals are more difficult to verify and noisy assessments can take place. This brings agents to have different evaluation of the new equilibrium price that corresponds to a divergence of (classical) probabilistic beliefs about payoff relevant signals. If the probability of the price increase and decrease is the same, then the price is in a short term equilibrium, until new informational signals reach the finance market, [23]. For the composite finance market one can observe the frequency of trading after the informational signal, e.g. if $S_{+}$, the company will pay the dividend with certainty. In an ideal case, if all the agents buy the asset (they hold singular beliefs and information update) the price goes up to the new equilibrium price. In this setting, the commutativity axiom is also satisfied, and no context effects (related to the order of information processing) are present, $p\left(P_{+} \mid S_{1} \cap S_{2}\right)=p\left(P_{+} \mid S_{2} \cap S_{1}\right)$, where $p$ denotes probability and $S_{1}, S_{2}$ some dividend signals. Next, under uncertainty the agents are able to evaluate instantaneously the past and present information and form subjective probabilities, associated with future signals and conditional price realizations. This mode of information processing can be formalized with the aid of the formula of total probability (FTP) which lies at the heart of classical probability theory.

In the general case the $P_{i}$ corresponds to the realization of a concrete price value, the $S_{k}$ corresponds some informational signal and $p$ is associated 
probability measure. ${ }^{13}$

$$
p\left(P_{i}\right)=\sum_{k=1}^{\infty} p\left(P_{i} \cap S_{k}\right)=\sum_{k=1}^{\infty} p\left(S_{k}\right) \times \frac{p\left(P_{i} \cap S_{k}\right)}{p\left(S_{k}\right)}
$$

We remind that the disjoint subsets $S_{k}$ and $P_{i}$ belong to the same probability sample space $\Omega$. A simple decision tree represents information processing of agents in classical probability framework.

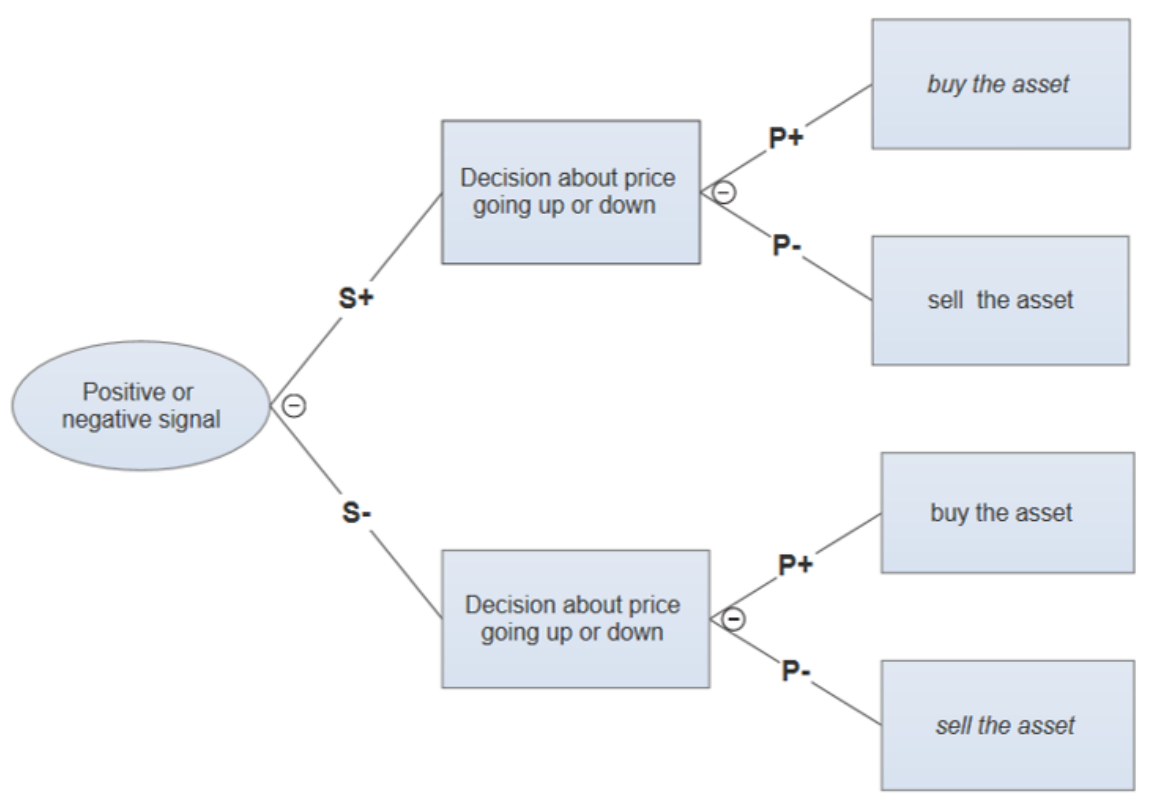

Figure 1: The chance nodes are given by circles and the belief/decision nodes are given by squares.

We can see from figure 2.1 that when a positive or negative signal reaches the market the belief states of agents update via Bayes rule, giving the conditional probability for $\left(P_{+} \mid S_{+}\right),\left(P_{+} \mid S_{-}\right),\left(P_{-} \mid S_{+}\right),\left(P_{-} \mid S_{-}\right)$. The conditional probabilities can be interpreted in Bayesian fashion as each agent's subjective beliefs about a price increase, given that $S_{ \pm}$is true. Naturally, an economically rational agent would always assume a $P+$ realisation, if the signal is positive and vice versa. ${ }^{14}$ Under a divergence of beliefs, given an objectivefrequency interpretation, one can observe that some populations of agents

\footnotetext{
${ }^{13}$ We operate with discrete probability measures to follow the formulation of classical neo-economic decision theories. We also restrict the formulation to dichotomous outcomes of variables, $P_{i}= \pm$ and $S_{k}= \pm$.

${ }^{14}$ We discuss in more detail the objective versus subjective interpretation of classical and quantum probability in Section 3).
} 
believe in e.g. $\left(P_{+} \mid S_{+}\right)$and less frequently in $\left(P_{-} \mid S_{+}\right)$. From here one can derive a divergence of beliefs based on classical probability that yields trading of the risky asset among these ensembles agents. If all agents hold the same beliefs about the fundamental asset price, given the set of informational signals, one would observe $p\left(P_{ \pm}\right)$to be equal to unity. The same mechanism applies, when the agents update their belief states in respect to the negative signal $S_{-}{ }^{15}$

With dichotomous signals and price realizations we get:

$$
p\left(P_{+}\right)=p\left(S_{+}\right) \times p\left(P_{+} \mid S_{+}\right)+p\left(S_{-}\right) \times p\left(P_{+} \mid S_{-}\right)
$$

for the price realisation $P_{+}$. The probability $(p)$ on the left-handside provides a probabilistic prognosis of the asset price increase (to a specific value) assuming a representative agent information processing under a set of different informational scenarios. Here we also assume that agents act upon their beliefs by maximizing the expected utility. In the same way, a total probability of $\left(P_{-}\right)$expectation can be expressed. Under the frequency interpretation, one can observe the frequency of agents that would hold or buy the unit of the asset for the next trading period, based on their firm beliefs.

\subsection{Possibility of a violation of the classical mode of information processing: quantum probability rep- resentation}

Extensive evidence on decision making under uncertainty and risk shows that agents often do not process information in classical probabilistic mode and do not employ Bayesian updating scheme. There are contexts, in which agents can process information in classical probabilistic mode, and contexts in which agents are not able, or prefer not to process information classically. Setting of uncertainty pertains to the non classical mode of information processing supported by empirical findings from psychology and behavioural economics, see a theoretical analysis and discussion in [2], [12], [26], [27], [47] and [48]. The above list of QP motivated works to information processing fallacies and preference reversals is far from being exhaustive. As documented by [51], and references herein, a real setting of the finance market is characterised by a vast level of information complexity and ambiguity, and hence agents'

\footnotetext{
${ }^{15}$ In a more general setup, a random variable $S$ can have multiple realizations (dividend values), upon which the agents condition the price outcomes. In classical finance models with continuous probability distribution of dividends, agents would possess a price function that maps each value of the dividend into a real value of a price at any time, $t$.
} 
decision making may not follow the cannons of classical probability based information processing.

QP has been shown to be able to describe the divergence in agents' posterior beliefs that are at variance with Bayesian inference and linearity of probability measures. The interference effects and the order of information processing can affect agents' limiting distribution of beliefs as shown in [47], [55], [33], and [10]. Ambiguous beliefs are well captured via probability interference that can create amplification of optimistic, or pessimistic expectations under ambiguity via probability wave interference.

In the quantum probability framework instead of a probability sample space, the price and fundamentals observables are represented in the complex Hilbert state space, and the events are given by subspaces. In the simplest two dimensional model, these are one-dimensional rays. A QP formulation relaxes the distributive axiom, where a joint distribution of price probabilities and dividend signals may not be accessible to the decision makers. Non-satisfaction of the commutativity condition of classical probability theory, as the order of information processing affects the final distribution of agents' beliefs, is given via incompatible observables in quantum probability setup. When price and dividend observables are not measured, the agent's belief state is an ambiguous state, in which different beliefs about signals and prices may coexist. It is only when the agents trade the asset based on their expectations, the measurements becomes classical Von Neuman-Lüders measurement, where the belief state collapses either into $P_{+}$or $P_{-}{ }^{16}$ Given that each agent is endowed at $t=0$ with $1 / N$ units of the risky asset $I$ and no liquidity constraints, the beliefs about the price states bring the acts to hold and buy, respective to sell. When the information is present on the finance market and it is verifiable, an ideal case from the viewpoint of EMH is that all agents buy an asset, until particular price threshold is reached, establishing a new unique REE. In that way no overreaction or under-reaction takes place by any ensembles of agents. Under divergence of beliefs, agents can be in different initial (quantum) belief states. The divergence in initial belief states can be caused by optimism, respective pessimism. Under the impossibility of short selling, the agents can: i) buy the asset, even if not able to resolve the uncertainty about the informational signals and price expectations in the next trading period, ii) overreact to the dividend signals based on their prior optimistic belief states about the asset price. More precisely, a classical FTP is replaced with a more general quantum formula of total

\footnotetext{
${ }^{16}$ On the real finance market the measurements can also be 'unsharp'. For instance, an agent is almost confident that she will trade an asset, given her assessment of the future price realization, yet some ambiguity is still present in her preference formation.
} 


\footnotetext{
${ }^{17}$ The theoretical propositions that are derived from the model can be tested empirically
}

probability (QFTP) that has an additional interference term that we denote by $\lambda$. A representation for a dichotomous outcome case:

$$
p\left(P_{+}\right)=p\left(P_{+} \mid S_{+}\right) p\left(S_{+}\right)+p\left(P_{+} \mid S_{-}\right) p\left(S_{-}\right)+2 \cos \theta \sqrt{p\left(P_{+} \mid S_{+}\right) p\left(S_{+}\right) p\left(P_{+} \mid S_{-}\right) p\left(S_{-}\right)}
$$

$$
\lambda=p\left(P_{+}\right)-p\left(P_{+} \mid S_{+}\right) p\left(S_{+}\right)-p\left(P_{+} \mid S_{-}\right) p\left(S_{-}\right)
$$

The parameter $\cos \theta \neq 0$ makes the whole term $\lambda$ either negative or positive. The quantum probabilistic formulation allows for the interference of agents' beliefs about signals and price realizations, giving raise to inflationary (the $\lambda>0$ ), or deflationary pressures (the $\lambda<0$ ) on the on asset prices under ambiguity. In mathematical language, we expect sub-additivity effects respective super-additivity effects of agents' beliefs about asset price states in the next trading period $t=1$. If $\lambda=0$ then QFTP collapses into FTP, and agents update the information in a classical mode under ambiguity. This means that no deviation from the fundamental value is observed. In several recent studies in economics and finance, the above mentioned interference angle $(\theta)$ was quantified experimentally and decision making contexts associated with its observed values were explored, cf.[47], [26], [2], [27]. The last work specifically explores preference formation in an investment context under risk, called a 'Portfolio Game'.

When agents are ambiguous but their initial (prior) belief states are identical, the total frequency of their trading under uncertainty can be approximated by the probability of price increase in the next trading period. The preference for buying under uncertainty is based on agents' belief interference of probability amplitudes related to $S \pm$, and $P \pm$. One can obtain the total probability of $P_{+}$, given by the left hand side of (2). If all agents hold time separable rational preferences, then the discounted expected value (E) $\sum\left(P_{t=1} \pm\right)$, gives a equilibrium price $P_{t=0}$. When the decision makers are in non-classical ambiguous belief states, $P_{t=0}$ can be different from the price based on classical information processing, (1). The interference term denoted in eq. (3) as $\lambda$, quantifies this difference. The variable $\theta$ denotes the angle of the belief state wave function of the agents. Constructive and destructive interference terms in the quantum probability framework explain the relationship between trading under uncertainty and the inflationary, respective deflationary pressures on the asset price. Interference in belief formation is shaped by incompatible decision making contexts, encountered by financial agents under uncertainty. ${ }^{17}$ We represent such a state update in Section, 4 
and extend the formulation in Appendix, 8. The above formalization assumes a representative agent (there is no divergence in probability interference angle and the initial belief states) and hence, a pure state representation can be used. When the agents are in different pure belief states they will have different probability interference magnitudes under ambiguity that are modeled as a composite (mixed) state of given by weighted sum of pure states, please see Section (3).

\section{$3 \quad$ Uncertainty versus diversity}

In this section we analyse in more detail the quantum versus classical representations of uncertainty and diversity of probabilistic beliefs. The difference can be perceived as subtle, yet we aim to draw a distinction between different subjective beliefs of agents as opposed to different ambiguous beliefs of agents on the possible values of the signal and the price. The below mathematical analysis is just the first step towards the understanding of this important distinction in asset trading context.

In the simplest quantum probability based model, market's state is based on the two dimensional qubit state space $H$. Consider in $H$ the orthonormal basis $|+\rangle,|-\rangle$, where $| \pm\rangle$ are the states representing the beliefs that the price of some infinitely lived financial asset will go up and down in the next trading period. Since uncertainty is present in respect to the outcome of the future distribution of dividends and prices, the agents hold ambiguous beliefs about the asset price states and hence, their trading under ambiguous beliefs generates deviations from the fundamental price value of the stock $I$.

Consider the state of the market before the arrival of the information about the value of some fundamentals.In a state of maximal ambiguity, the pure state representation of the beliefs of a representative agent are given as the uniform superposition of the price states, respective dividend states.

$$
|\psi\rangle=\frac{|+\rangle+|-\rangle}{\sqrt{2}} .
$$

This is the state of a maximal uncertainty of market's agents about the future dividend raise/fall and the consequent price raise/fall. If the agents possess some prior information (e.g. observed realization of previous divided and price outcomes), they can make stronger expectations about the price would go up or down, and hence the superposition state would be be not

by the measurement of interference terms from the limiting probabilities and belief state reconstruction via the Born rule. 
uniform, where the squared modulus of the complex state coordinates $c, d$ would sum to unity and each provide a classical probabilistic outcome on the price state $( \pm)$.

$$
|\psi\rangle=c|+\rangle+d|-\rangle, c, d \in \mathbf{C},|c|^{2}+|d|^{2}=1 .
$$

\footnotetext{
${ }^{18}$ This is a fundamental remark that separates the proposed model from the information asymmetry based models, where diversity of expectations is encoded in different prior and posteriors probability distributions that agents possess over the asset valuation.

${ }^{19}$ Future venues of research can focus on a generalization of the model to a market portfolio of assets, where the agents form expectations about the price behaviour of the composite finance market dynamics, see discussion in [51] and theoretical contribution in $[34]$.
} 
ensemble of agents $\Omega$ is split into two sub-ensembles $\Omega_{A}$ and $\Omega_{B}$ characterizing that agents belonging to each of them have the same state of uncertainty about possible behavior of the price.

In quantum probability random variables are given by observables, hence we introduce the price expectations observable, $P$, for the asset $I$ represented by the operator having the vectors $| \pm\rangle$ as eigenvectors with eigenvalues \pm 1 :

$$
P=|+\rangle\langle+|-|-\rangle\langle-| \equiv P_{+}-P_{-} .
$$

Before forming a concrete preference on holding, or selling the unit of an asset, an agent has to resolve her ambiguity about the possible behavior of the stock price realization. For the simplicity of the model, the preferences are given classically, based on the discounted expected future value of stock price in the next trading period. To resolve the (non-classical) ambiguity the agent performs a (self-)measurement of the corresponding price expectation observable represented by the operator $P$. This operator, jointly with agent's belief-state $\psi$ that is in a superposition of the different informational signals given as in eq. (5), encodes the probabilities that the price of this asset will go up or down. ${ }^{20}$ They are given by Born's rule (one of the core postulates of quantum mechanics):

$$
p( \pm)=|\langle \pm \mid \psi\rangle|^{2}=\left\|P_{ \pm} \psi\right\|^{2} .
$$

For a pure state Born rule normalizes the quantum measurement scheme on an observable. More specifically, it identifies the probability rule for observing probability of a realization of an eigenstate (price value) after the measurement of the price behaviour observable $P$.

For a mixed state with a density matrix $p$, Born rule can be expressed via a trace formalism:

$$
p( \pm)=\operatorname{Tr} \rho P_{ \pm}
$$

In this setup the limiting distribution of obtaining a concrete price output \pm for asset $I$ in the next trading period is given by taking the trace of the action of a projector $P_{ \pm}$upon the mixed state $\rho$.

One can measure the heterogeneity of beliefs about the asset liquidation value at $t=1$, by considering, beliefs of the ensembles of agents $A, B \in \Omega$ separately, in a similar mode as in [50]. ${ }^{21}$ If one cohort of agents is in the pure state $\psi_{A}$ and the other is in the pure state $\psi_{B}$, the difference in

${ }^{20}$ To exemplify such a state transition, for the moment, we employ pure states.

${ }^{21}$ The dynamics of the value of belief heterogeneity denoted by $k$ can be in their model crucial for creating upwards volatility in asset prices, if $k>y \sigma^{2} Q$, where $Q$ denotes the total supply of the asset and $y$ is a standard measure of risk aversion. 
ambiguous beliefs is obtained my measuring the angle (phase) between the state vectors $\psi_{A}$ and $\psi_{B}$, such that $\theta_{A-B}=\Delta_{A B}$. If the ensemble $A$ holds exactly the opposite ambiguous beliefs to the ensemble $B$, then $\Delta_{A B}=\pi$.

\subsection{Interpretation of agents' beliefs: subjective and objective models}

In quantum probabilistic models to decision making there are explored two basic models for completion of the process of decision making by an agent. These models are based on the two core interpretations of probabilities, $a b$ jectivist and subjectivist. ${ }^{22}$

- Objective probability: an agent actually performs a measurement on one of the outcomes, \pm 1 , and depending on this outcome she makes her decision. The probabilities given by eq.(8) are objective (frequency) probabilities. They do not have any meaning for an individual agent. They can be found by collecting statistics for a large ensemble of agents. Such probability is interpreted as statistical frequency of agents that expect the asset to up or down.

- Subjective probability: an agent assigns subjective probabilities to possible outcomes given by eq. (8) and then she proceed as in the classical subjective decision making framework by calculating the odds and making her choice by comparing the odds with 1 (certainty). Subjective probability approach is also known in economic literature as the 'degree of belief'. In this paradigm one treats the probability as individual agents' beliefs about the realisations of dividend and price outcomes.

The second interpretation seems to be closer to the classical subjective probability models of decision making, such as SEUT and its non-linear generalisations. Moreover, the behavior of such an agent can be considered to be more rational from the viewpoint of neo-classical economic theory (see also analysis in section 2.1.) For instance, if the probability of a price decrease is unlikely, $p(P-)<<1$, then it is rational to make the decision that the price is to go up. Thus, an agent with the belief-state encoding very low probability that the price is to go down never expects that the price will go down and acts accordingly. This interpretation of expectation formation also allows to operate with the notion of a 'representative agent', assuming that

\footnotetext{
${ }^{22} \mathrm{~A}$ discussion on the interpretation of subjective and objective probability in experiments is provided in an early work by [54], for applications of QP interpretation in decision theory see [26].
} 
all agents have similar subjective beliefs and form identical preferences. In contrary, an objective probability, or frequency interpretation implies that single agents have different trading preferences. Of course, the probability (the frequency of agents, who have some certain beliefs and trade upon them) of such a decision is very low, but not zero, as in the subjective interpretation model. It might be that the validity of these two models can be tested experimentally. In fact, such an experimental comparison is not just a 'quantum probability theoretic problem'. This is a problem of application of objective versus subjective probabilities in models of decision making for populations of agents.

\subsection{State dependence in asset trading and feedback reaction}

How do the observed price states feed back into the ambiguous belief states of the agents about the asset price in the next trading period?

By making the decision $\alpha_{t}= \pm 1$ for the asset $I$ at time $t$ (which is given by $t=0$ in the first trading period), an agent's initial state $\psi$ is projected onto the eigenvector $\left|\alpha_{t}\right\rangle$ that corresponds to an eigenstate for a particular value of price realization for that asset in the current trading period. ${ }^{23}$ After the price realization up to time $t$ of the asset is observed, the agent has to make a decision about the possible price behavior of the asset at time $t+1$, and she performs a measurement of the corresponding expectation observable, for the updated belief-state $\left|t_{t+1}\right\rangle$. The index $t+1$ denotes agent's ambiguous beliefs about the dividends-prices in the subsequent trading period. The eigenvalues $\alpha_{t}= \pm 1$ of the price behaviour observable $P_{t}$, are given with the probability:

$$
p_{t \rightarrow(t+1)}\left(\alpha_{t} \rightarrow \alpha_{t+1}\right)=\left|\left\langle\alpha_{t} \mid \alpha_{t+1}\right\rangle\right|^{2} .
$$

The above mathematical exposition of state transition provides quantum transition probability. They have also an objective meaning. Consider an ensemble of agents in the same state $\psi$ who made the decision $\alpha_{t}$ with respect to the price's behavior of the asset. In the next step the agents form preferences about the subsequent period asset price realizations and consider only those whose decision is $\alpha_{t+1}$. In this way it is possible to find the frequency-probability $p_{t \rightarrow(t+1)}\left(\alpha_{t} \rightarrow \alpha_{t+1}\right)$. Following a classical tradition, we can consider the above output as the quantum analogue of the

\footnotetext{
${ }^{23}$ In a simple setup with two types of price movements we fix only two eigenvectors $\left|\alpha_{+}\right\rangle$ and $\left|\alpha_{-}\right\rangle$, corresponding to the eigenvalues $a= \pm 1$. These price outcomes are observed by the agent on the finance market when trading takes place.
} 
conditional probabilities, $p_{t \rightarrow(t+1)}\left(\alpha_{t} \rightarrow \alpha_{t+1}\right) \equiv p_{t+1 \mid t}\left(\alpha_{t+1} \mid \alpha_{t}\right)$. We remark that the trading in this setup takes place under informational ambiguity in respect to the next trading period, when the agents are still in an indeterminacy given as a superposition in respect to next coming dividend signals, $p(S \pm), \psi_{t}=\beta_{1}|+\rangle+\beta_{2}|-\rangle,\left|\beta_{1}\right|^{2}+\left|\beta_{2}\right|^{2}=1$. Hence, in each of the subsequent updated belief states about price behaviour the agents are in superposition in respect to the fundamentals that can change the price and interference effects as in eq.(2) exist for each agent's pure belief state (that can be approximated by a type of a representative agent). By using the probabilities (8)-(10) we can define the quantum joint probability distribution for price expectation about the price of the asset $I$ in both trading periods, $t$ and $t+1$.

$$
p_{t,(t+1)}\left(\alpha_{t}, \alpha_{t+1}\right)=p_{t}\left(\alpha_{t}\right) p_{(t+1) \mid t}\left(\alpha_{t+1} \mid \alpha_{t}\right) .
$$

This joint probability respects the order structure of beliefs, where the observed price outcome at time $t$ changes the beliefs about the asset price distribution at $t+1 .{ }^{24}$ In general:

$$
p_{t,(t+1)}\left(\alpha_{t}, \alpha_{t+1}\right) \neq p_{(t+1), t}\left(\alpha_{t+1}, \alpha_{t}\right) .
$$

Equation, (12) is an exhibition of the order effect that is not in accord with Bayesian probability updating scheme, see theoretical analysis in [48], [55]. Order effects bring a non-satisfaction of the joint probability distribution and give raise to violation of the commutativity principle of classical probability. Order effects in a state update under ambiguity can exist for: i) preference formation related to a sequence of asset price observation as depicted above; ii) information processing related to the order of the sequences of state updates from observed dividend signal realizations. Non-commuting observables allow to depict agents' state dependence in belief formation that affects their trading preferences and hence the RE equilibrium price departures. ${ }^{25}$ If state dependence is absent, the observable operators are commuting and the agent possesses a joint probability distribution for the infinite sequence of decision variables, given by some element, $\omega \in \Omega^{t}, \omega=\left\{P_{t}, S_{t}\right\}_{t=0}^{\infty}$.

It is important to remark that in the general quantum probability setup the operators for stock price behaviour at different time points do not commute, i.e., $\left[P_{t}, P_{t+1}\right] \neq 0$. This means that the price (and dividend signal)

\footnotetext{
${ }^{24}$ The same state update scheme takes place in respect to the informational signals, i.e. the state update in respect to the price implies that the bases associated with the dividend realizations have a different phase in respect to this new updated state at $\psi_{t}$. The limiting probability distribution of the asset prices, when current prices and dividends at time $t$ are observed is determined by agents' order of measurement of the corresponding observables.

${ }^{25}$ In the formalization above we focus on dependence of the belief stes upon the realised price states.
} 
observables overtime, are complementary and agents cannot form a joint probability space of these random variables in the process of information processing. The order of price and dividend observations creates the pessimistic, respective optimistic belief states of the agents that deviate from the classical joint evaluation of past and future price and dividend realizations. The important consequence is that it is impossible to define a family of random variables for dividends and prices denoted as $\xi_{i}: \Omega \rightarrow\{ \pm 1\}$ on the same classical probability space, $(\Omega, \mathcal{F} ; P)$, which would reproduce the quantum probabilities $p( \pm 1)=|\langle \pm \mid \psi\rangle|^{2}$ as $P\left(\xi_{i}= \pm\right)$ and quantum transition probabilities $p_{t \rightarrow(t+1)}\left(\alpha_{t} \rightarrow \alpha_{t+1}\right)=\left|\left\langle\alpha_{t} \mid \alpha_{t+1}\right\rangle\right|^{2}, \alpha_{t}, \alpha_{t+1}= \pm$, as the classical conditional probabilities $P\left(\xi_{t+1}=\alpha_{t+1} \mid \xi_{t}=\alpha_{t}\right)$.

In QP model the agents do not form definite expectations about the price behavior, and the observed price realization can change their future expectations about the asset price. This type of state dependence in beliefs is not in accord with classical RE pricing models that imply a current price dependence only on the future discounted payoff relevant variables. Furthermore, the agents exhibit ambiguity in respect to the probabilistic composition of future dividend signals and impact on price value, whereby the interference of these beliefs gives raise to a deviation of the belief distribution from the classically modeled rational beliefs. Given a price observation, agents can form conditional expectations only sequentially and not jointly. In the next section we present an operational depiction of the heterogeneous asset price belief evolution under informational ambiguity.

\section{Creation and annihilation operators}

We present a belief state space construct based on two ensembles of representative agents in different initial belief states and describe the operators that create their beliefs about the price of the risky asset. Consider a type $A$ agent, and her belief-state space $K$, about the price behaviour let it be a two dimensional qubit state space. ${ }^{26}$ We define an orthonormal basis in $K$, denoted as $|0\rangle,|1\rangle$. The states are interpreted as follows: they represent, respectively, agent $A^{\prime} s$ beliefs that the price of the stock will decrease or increase under ambiguity. In general $A$ is in a state of a superposition of these 'core beliefs' representing her ambiguity in respect to the asset price

\footnotetext{
${ }^{26}$ As noted above, the preference states of agents are visible from the market data, where the belief states about price behaviour are playing a key role, since the agents trade upon their beliefs, when there are no liquidity constraints. We introduce two operators $A$ and $B$ that describe the interaction of $A^{\prime} s$ beliefs about the price behaviour and the actual finance market price behaviour.
} 
configuration at $t+1$ :

$$
|\psi\rangle=c_{0}|0\rangle+c_{1}|1\rangle
$$

where $c_{j} \in \mathbf{C}$ and $\left|c_{0}\right|^{2}+\left|c_{1}\right|^{2}=1$.

The initial state $\psi$ encodes all price and dividend realization histories and encodes the prior ambiguous state of an agent. Two ensembles of agents that we introduce later, can have different states $\psi$ that also change over the run of trading periods. Mathematically, weighted combinations of the pure states, mixed states, are employed.

Following [35] we introduce the so called creation and annihilation operators $a^{\star}, a$, having the following role in the setting of asset trading. ${ }^{27}$

The operator $a^{\star}$ 'creates' a belief that the price would go up, $a^{\star}|0\rangle=|1\rangle$, and the operator $a$ 'annihilates' the belief that the price would go up, $a|1\rangle=$ $|0\rangle$. It can be interpreted as the operator of the creation of a belief that the the price is to go down. Hence, these operators provide a mathematical tool for the generation of firm beliefs about the price change. These operators satisfy canonical anti-commutation relations:

$$
\left\{a, a^{\star}\right\}=I,\{a, a\}=0,\left\{a^{\star}, a^{\star}\right\}=0,
$$

where $I$ is the unit operator and $\{A ; B\}=A B+B A$ denotes anti-commutator of two operators $A, B$. In the basis $|0\rangle,|1\rangle$ the operators can be represented by $2 \times 2$ matrices:

$$
a^{\star}=\left(\begin{array}{cc}
0 & 0 \\
1 & 0
\end{array}\right), a=\left(\begin{array}{ll}
0 & 1 \\
0 & 0
\end{array}\right) .
$$

We now introduce the operator of the price behavior $B=a^{\star} a$. We remark that $B|1\rangle=|1\rangle$ and $B|0\rangle=0$. Thus, in the basis of sharp beliefs about the price behavior $B$ has the diagonal form $B=\operatorname{diag}(0,1)$. This operator, in fact, coincides with the orthogonal projector onto the vector $|1\rangle$, i.e., $B=|1\rangle\langle 1|$. This operator represents the observable of the actual price behavior on the finance market. Agents can make self-inspections of their beliefs about the

${ }^{27}$ The paper by [6] apply raising a lowering operators to describe the process of creation and reduction of traders' stock holdings. See also the work by [7] that uses this formulation to describe trading between two agents, or a system of ' $\mathrm{n}$ agents in a general trading game. The operators can be applied to describe non classical dynamics in more complex macroscopic systems, [8]. An interpretation of raising and lowering operators in our framework is given by their operational role in modeling the price changes in financial markets, while belief update of agents takes place. 
possible price changes throughout the investment process. ${ }^{28}$ By applying the inverse Born's rule one can reconstruct the subjective probability distribution of the dividend signals from agents' initial belief states coupled with the observed price states, given that $P_{t}=e^{-r \Delta t}\left(\left|c_{1}\right|^{2} P_{+}+\left|c_{2}\right|^{2} P_{-}\right)$. The squared complex coordinates $c_{1}, c_{2}$ denote the total probability of the price increase or decrease given by eq. (2).

In this set-up an agent that is isolated from the surrounding informational environment, can observe her trading preferences and the uncertainty that can be updated via her observations of the asset price behaviour. We remark that the observed asset prices can further enhance the agent's $A$ optimism in respect to the realization of future positive signals and price outcomes, as depicted in Section, 3.2.

Now we consider the system of two types of agents $(A, B)$ with beliefstate spaces $K_{i}, i=1,2$, with bases $|0\rangle_{i},|1\rangle_{i}$. The belief-state space of this system is given by the tensor product $K=K_{1} \otimes K_{2}$ and it has the basis $|00\rangle,|10\rangle,|01\rangle,|11\rangle .{ }^{29}$ The basis states $|\alpha \beta\rangle$ are the states of sharp beliefs, e.g., in the state $|00\rangle$ both agents believe with certainty that the price of the financial asset will go down.

The individual ambiguity of the agents is encoded in superposition of the form in eq.(13). The joint belief-state of two agents is given by the factor product:

$$
\left|\psi_{1}\right\rangle \otimes\left|\psi_{2}\right\rangle=\left(c_{0}|0\rangle+c_{1}|1\rangle\right) \otimes\left(k_{0}|0\rangle+k_{1}|1\rangle\right)
$$

The most general belief-state of these two agents has the form of superposition:

$$
|\psi\rangle=c_{00}|00\rangle+c_{10}|10\rangle+c_{01}|01\rangle+c_{11}|11\rangle
$$

where $c_{i j} \in \mathbf{C}$ and $\left|c_{00}\right|^{2}+\ldots+\left|c_{11}\right|^{2}=1$.

The creation and annihilation operators of agents are lifted to the beliefstate space $K$ and we denote them by bold symbols, e.g., $\mathbf{a}_{1}=a_{1} \otimes I$. These operators satisfy so called qubit commutation relations. For the fixed $i$, such operators satisfy the canonical commutation relations, see eq.(14) for the one dimensional fermionic system, but for different $i, j$ they commute:

$$
\left[\mathbf{a}_{i}, \mathbf{a}_{j}^{\star}\right]=\left[\mathbf{a}_{i}, \mathbf{a}_{j}\right]=\left[\mathbf{a}_{i}^{\star}, \mathbf{a}_{j}^{\star}\right]=0,
$$

where $[A, B]=A B-B A$ is the usual commutator.

\footnotetext{
${ }^{28}$ In the work by [14] a price function of a form $p(s)$ is introduced, which maps the signals into asset prices, and the agents can infer the probability distributions from the price function by taking its inverse. We do not directly associate multiple signals (a set of different signals) with the observed price, since the beliefs about the signals are ambiguous.

${ }^{29}$ Here we simplified notation, $|\alpha\rangle_{i} \equiv|\alpha\rangle$ and $|\alpha\rangle_{1} \otimes|\beta\rangle_{2} \equiv|\alpha \beta\rangle, \alpha, \beta=0,1$.
} 
For the composite state of the two agent types the introduced operators generate agents' joint belief dynamics and hence, give the price expectations associated with the composite set of agents that trade the risky asset.

\section{The Born rule of information update}

After formulating the belief state evolution process with the aid of the pricecreation and annihilation operators, the next stage is to explain how the ambiguity of agents' beliefs is resolved to classical probabilistic distribution of belief states after arrival of signals. By this we mean how: a) each agent's belief state and, b) a mixture of agents' belief states giving the composite finance market will update once a signal about asset prices reaches the market. The probability of state realization and conditional probabilities of signals are given by Born rule (for mathematical details cf. monographs [12], [26]. ${ }^{30}$ Born rule specifies probability to obtain a particular result of measurement (eigenvalue) after a measurement on a pure state $\psi$, or mixed state given by a density matrix $\rho$. The formulation of Born rule differs for pure and mixed states, yet in both cases it specifies the classical limiting probability distribution associated with each eigenvalue realization. We specify the dividend signal observable $\lambda$ with a corresponding operator $S$ that has dichotomous eigenvalues \pm 1 , with $\left| \pm_{k}\right\rangle$ as its eigenvectors.

As specified in eq.(8), we can observe the probability of arrival of some new dividend signal for the pure initial belief state $\psi$ as:

$$
p\left(S_{ \pm}\right)=\left\|S_{ \pm} \psi\right\|^{2} .
$$

We can in the similar way introduce $\mu$ as the price movement observable with corresponding operator, $P$ that is now measured after the arrival after dividend outcome \pm .

The operator has eigenvectors $| \pm\rangle$. Conditional probability from eq. 20 specifying the probability of obtaining a price value \pm , under the condition that signal \pm was observed is given in a similar way as in eq.(10). We denote here the signal and price operator eigenvectors via $\lambda_{ \pm}$respective $\mu_{ \pm}$ to elucidate the state transition scheme from one (normalized) eigenvector to another:

$$
p\left(P_{ \pm} \mid S_{ \pm}\right)=\left|\left\langle\lambda_{ \pm} \mid \mu_{ \pm}\right\rangle\right|^{2}
$$

The conditional probability in eq.(20) contains the information about the operator $S$ that updates the price behaviour and as a result trading preferences, after an information arrival, such as some signal. Moreover, the conditional

\footnotetext{
${ }^{30}$ Probabilities can be subjective or objective, as discussed in Section 3.
} 
probability will contain the information regarding interaction between the observables $\mu$ and $\lambda$ acting upon the initial ambiguous belief state of financial agents. ${ }^{31}$ In a real finance market setting the agents can also have an internal dynamics of their ambiguous beliefs in addition to the updates of the $S, P$ as well as the ambiguity in respect to these observables may not be resolved concurrently. In the Appendix, 8 we devise a more detailed mathematical representation of agents information processing and measurement scheme about the price behaviour.

The order of information (or the order, in which the agent chooses to process the information) alike the choice on asset trading exposed in Section 3 can affect her updated belief state. Order effects can be modeled in QP via different eigenvectors associated with the observables and hence, the effect of phase between the eigenvectors upon the final conditional probabilities under different sequences of measurement schemes surfaces. We recap that he impact of measurement sequence upon conditional probabilities is due to incompatible observables in this setup. The order effect is important to understand the state update under different orderings of information that can create posterior biased belief state of overoptimism, respective pessimism. The effect of such state updates via Born rule (the sum of which is given by the composite market mixed state) can give raise to sudden price behaviour changes, due to interference effects of information and action states. Such a process can be captured via dynamical quantum probabilistic models, see [47], [28], [12], [26], [34], [40] applying the Schroedinger equation and its extensions to model the belief and information dynamics.

\section{Discussion: empirical predictions?}

Our model so far has described the quantum probabilistic formulation of the uncertainty, or divergence of belief states of agents that creates asset price volatility in the capital market. The central contribution of this work is due to formalization of the of divergence of beliefs in classical versus quantum probabilistic framework given ambiguity about both dividend and price states shaped by agents internal states and informationally incomplete markets. The motivation to apply QP is to capture agents' trading under a deeper, endogenous uncertainty.

However, for empirical prediction of the model we need to consider how the measurements of belief states performed by the signal and price behaviour

\footnotetext{
${ }^{31}$ The QP update algorithm allows also to depict information update from uninformative and close to zero priors that cannot be captured by classical Bayesian update, see the work by [10].
} 
operators on the initial belief states of the agents, or the market belief state as a whole, impacts the probabilities of movements of asset prices. Certainly, according to QP formulation the probabilities are obtained from the familiar trace formulation, as shown in the above model, which contains the interference terms for different ensembles of agents. Since the size of the interference term indicates the magnitude of probabilistic interference, we can surely talk about e.g. sub-additivity of baseline probability for $P_{+}$, if it is above the sum of the conditional probabilistic disjunctions given the different signals (the so called 'disjunction effect' is present). On the aggregate finance market, we can interpret it as a bubble in the condition of uncertainty, when the actual signals are not measured by market participants in a Bayesian fashion. When interference effect is positive, an overweighting of probability takes place, and the $p\left(P_{+}\right)$is above the total probability of $P_{+}$, given the different sets of information as specified in FTP.

As noted, when $\cos \theta=0$ no interference is present and QFTP collapses into its classical analogue the FTP so that agents have a classical probabilistic distribution of the asset price expectations, given the different signals. In this case all agents agree on the fundamental value, given that there is no dispersion in their information processing. A positive, respective negative magnitude of the interference term also depends on the belief evolution dynamics under uncertainty, and periods of 'optimistic', respective 'pessimistic' trading cycles can emerge, where bubbles can burst suddenly, without any warning signals, [56]. We remark that in contrary to the modifications of classical probability calculus that are introduced to describe volatility cycles form agents' beliefs in the classical finance literature, the endeavour of QP based framework of asset trading is to consider a different probability calculus that is complete in terms of its axiomatics.

Since the two ensembles of agents can have different belief states about the price ups and downs, the whole market can be given mathematically a system of these ensembles of agents in different pure belief states, denoted via a mixed state. This representation allows to depict divergence of the uncertain (pure) belief states of agents, where under short selling constrains agent ensembles with positive $\lambda$ create inflationary pressures. The model generalized to markets with no short selling constraints, makes the pessimists and also speculators act by creating periods of asset undervaluation, whereby the evolution of the mixed state in the QP model allows to observe the net effect on the limiting probability distribution of the price states, given in eq.(9). The proposed theoretical model can be advanced further, by replacing the notion of risk neutral agent and introducing a discount factor based on the risk aversion of the different ensembles of agents, see [1].

Finally, the QP based model of beliefs has the potential to provide man- 
agers and practitioners with an insight into the possible response patterns of investors to new share issuance as well as valuation of the traded risky assets on the secondary markets. The studies can be based on the empirical investigations of interference terms and of dispersion of opinions data as mentioned above. Such studies would bring more insights about the future expectations of primary and secondary markets of risky assets. The probabilistic prognosis of agents' price valuation under the subjectivist probability interpretation can allow for better understanding of cognitive processes of individual investors as attested experimentally in, e.g. [27], [37]. The 'objectivist' interpretation of the quantum probabilities would correspond to the prognosis of the frequency of agents buying and selling the asset under ambiguity and hence, provide an indication of the composite finance market trading volume.

\section{Conclusion}

We have suggested a QP based model of asset trading behaviour under uncertainty. Our model fundamentally differentiates from the standard neoclassical finance models of price behaviour under heterogeneous beliefs, based on classical probability theory and non-additive modifications. The main motivation for adopting an alternative modeling is that there is a high degree of divergence in predictions in the standard literature regarding the price behavior under uncertainty, for example, whether diversity of beliefs leads to adverse selection problem, or overpricing respective under-pricing following speculative trading. There is also a lack of a unifying framework describing the mechanism of speculative bubble formation but also trading that can lead to market crashes. Our model aims to offer an alternative foundation to speculative asset pricing under ambiguity, based on QP of belief representation. First of all, the description of uncertainty in the model is based on a superposition of belief states, and not on classical probability distributions. We also deploy a novel technique of anhilliation-creation operators, to describe observables that measure the "belief state" of the market. The interference of probability amplitudes related to price states and fundamentals gives a measure of over-pricing and in other trading periods under-pricing, following the state dependence on observed asset price states. The proposed framework in the subjectivist probability interpretation, is providing a quantifiable testable prediction on price volatility in respect to the from fundamental value, under belief ambiguity. The framework can be further tested in experimental asset trading markets, where one can reckon the degree of inflationary, or deflationary pressures created by the ensembles of traders in different be- 
lief states under ambiguity. The agents' asset trading preferences can be revealed under uncertainty and contrasted with their preferences after some informational signals reached them to attest the classicality of their belief update in investment, as performed in a similar setting in [27]. Order effects belief evolution, given by the order of the observed price states and payoff relevant variables can be also further tested with a similar setup as recently proposed in [40]. We hope that this simple theoretical model will bring up new experimental studies in the area of investment preferences of agents for different types of financial instruments and financial markets, coupled with the impact of stock market news upon the evolution of agents' expectations that can be potentially modeled in a QP based decision theoretic framework.

\section{Appendix}

We espouse a more detailed mathematical representation of the existence of internal dynamics of the belief states of the agent types, given by their divergent mode of information processing, beyond the measurements of dividend and price signals at the specific points in time $\left(t_{0}-t\right)$.

Without loss of generality, let us consider a Hilbert state space $H$ of an arbitrary dimension, in which two operators $S$ and $P$ with respective eigenvalues \pm 1 and eigen-subspaces $H_{ \pm}^{S}$ and $H_{ \pm}^{P}$, act upon the preference state of the agents. The corresponding projectors are denoted by $S_{ \pm}$and $P_{ \pm}$, i.e., $S=S_{+}-S_{-}$and $P=P_{+}-P_{-}$.

The measurements of $S$ (or $P$ ) for the state $\psi$ with the outcomes \pm 1 projects $\psi$ onto the state $\psi_{ \pm}^{S}=S_{ \pm} \psi\left(\right.$ or $\left.\psi_{ \pm}^{P}=P_{ \pm} \psi\right)$. The belief state of an agent can be modified (updated) through the measurements of $S$ or $P$. This is basics of the (belief) measurement scheme due to the 'Von Neuman-Lüders' projection postulate of quantum theory.

It is useful to extend exposition of quantum probabilistic belief update to include the internal dynamics of the belief state $t \rightarrow \psi_{t}$ in order to better approximate the information processing to real finance market environment. This is a belief dynamics of agents in the absence of the updates, given by the measurements of $S$ and $P$ observables. Such dynamics is mathematically described by a family of unitary operators $U_{t}$ that transform the ambiguous belief distribution overtime, where $\psi_{t}=U\left(t-t_{0}\right) \psi_{0}$.

Suppose that at instances of time $t_{1}, \ldots, t_{n}, \ldots$ the agent performs a measurements of $S$, or in other words she gets a signal that dividends will raise or fall, and at instances of time $s_{1}, \ldots, s_{n}, \ldots$ she measures $P$, so she updates her expectations about the asset price based on the observed price dynamics. It natural to assume that $t_{1}<s_{1}<t_{2}<\ldots<t_{n}<s_{n} \ldots$ since the agents 
trade under informational ambiguity.

Hence a complete dynamics of an agent's belief state can be represented as a series of projections coupled with the (agent specific) unitary evolution:

$$
\psi_{t_{0}} \rightarrow \psi_{t_{1}}=\frac{S_{\lambda_{1}} U_{t_{1}-t_{0}} \psi_{t_{0}}}{\left\|S_{\lambda_{t_{1}}} U_{t_{1}-t_{0}} \psi_{t_{0}}\right\|} \rightarrow \psi_{s_{1}}=\frac{P_{\mu_{s_{1}}} U_{s_{1}-t_{1}} \psi_{t_{1}}}{\left\|P_{\mu_{s_{1}}} U_{s_{1}-t_{1}} \psi_{t_{1}}\right\|} \rightarrow \ldots,
$$

where $\lambda_{t_{j}}= \pm 1$ and $m_{s_{i}}= \pm 1$ are the outcomes of the measurements of $S$ and $P$, respectively.

For any $t$, the probabilities of the possible measurements outcomes $S$ and $P$ are given by the Born rule: $p(S= \pm 1)=\left\|S_{ \pm} \psi_{t}\right\|^{2}$ and $p(P= \pm 1)=$ $\left\|P_{ \pm} \psi_{t}\right\|^{2}$. In particular, for an instance of time $t_{j}$, we obtain $p\left(S=\lambda_{t_{j}}\right)=1$, for the next instance of time $s_{i}$, we obtain $p\left(P=m_{s_{i}}\right)=1$.

The model also specifies transition probabilities, e.g., the probability of transition from belief $\lambda_{t_{j}}$ about the dividend distribution at $t=t_{j}$ to a belief $\mu_{s_{j}}$ about the price distribution: $p\left(\lambda_{t_{j}} \rightarrow \mu_{s_{j}}\right)=\left|\left\langle\psi_{s_{j}} \mid \psi_{t_{j}}\right\rangle\right|^{2}$.

In the simplified model that we proposed earlier, we omit the impact of the internal evolution of the belief state, i.e., to set $U_{t} \equiv I$. Here the state dynamics is reduced to a series of state updates resulting from the measurements of the core variables $S$ and $P$ :

$$
\psi_{t_{0}} \rightarrow \psi_{t_{1}}=\frac{S_{\lambda_{t_{1}}} \psi_{t_{0}}}{\left\|S_{\lambda_{t_{1}}} \psi_{t_{0}}\right\|} \rightarrow \psi_{s_{1}}=\frac{P_{\mu_{s_{1}}} \psi_{t_{1}}}{\left\|P_{\mu_{s_{1}}} \psi_{t_{1}}\right\|} \rightarrow \ldots,
$$

Thus, for $t_{j} \leq t<s_{j}$, we get $\psi_{t}=S_{\lambda_{t_{j}}} \ldots P_{\mu_{s_{1}}} S_{\lambda_{t_{1}}} \psi_{t_{0}} /\left\|S_{\lambda_{t_{j}}} \ldots P_{\mu_{s_{1}}} S_{\lambda_{t_{1}}} \psi_{t_{0}}\right\|$, and for $s_{j} \leq t<t_{j+1}$,

$$
\psi_{t}=P_{\mu_{s_{j}}} S_{\lambda_{t_{j}}} \ldots P_{\mu_{s_{1}}} S_{\lambda_{t_{1}}} \psi_{t_{0}} /\left\|P_{\mu_{s_{j}}} S_{\lambda_{t_{j}}} \ldots P_{\mu_{s_{1}}} S_{\lambda_{t_{1}}} \psi_{t_{0}}\right\| .
$$

The sources of internal dynamics can be manifold and are given by agent specific variables. Some salient triggers of agent specific belief evolution detected experimentally [37], [40] due to individual differences in learning from own gains and losses as well as decision outcomes, coupled with agent's cognitive capacity and risk attitude.

\section{Acknowledgements}

We would like to thank Emmanuel Haven, Christoph Gallus and the participants of the special session on 'Complementarity Beyond Physics: Quantum reasoning in philosophy, psychology and economics' at the UQT Conference in Vaxjo, 2018 for their constructive comments. We also would like to thank 
the participants of the International Cross Disciplinary Conference on Cognitive Technologies and Quantum Intelligence, ITMO, St. Petersburg, 2018, for helpful comments and suggestions. The comments and suggestions of the referees and the editor significantly improved the paper. We are solely responsible for any errors.

\section{References}

[1] Adam, K., Marcet, A. 2011. Internal Rationality, imperfect market knowledge and asset prices. J. Econ. Theory, 146, 1224-1252.

[2] Aerts, D., Haven, E., Sozzo, S., 2017. A Proposal to Extend Expected Utility in a Quantum Probabilistic Framework. Econ. Theory, DOI: 10.1007/s00199-017-1051-2.

[3] Ausloos, M., Jovanovic, F. Schinckus, C. 2016. On the "usual" Misunderstanding between Econophysics and Finance: Some Clarifications on Modelling Approaches and the Efficient Market Hypothesis. Int. Rev. Fin. Analysis. 47, 7-14.

[4] Baaquie, B.E., 2004. Quantum Finance: Path Integrals and Hamiltonians for Options and Interest Rates. Cambridge University Press.

[5] Baaquie, B.E., 2009. Interest Rates and the Coupon Bonds in Quantum Finance. Cambridge University Press.

[6] Bagarello, F., 2006. An operational approach to stock market. J.Phys. A. $39,6823-6840$.

[7] Bagarello, F. , Haven, E., 2016. First Results on Applying a non-linear Effect Formalism to Alliances between Political Parties and Buy and Sell Dynamics. Physica A:Stat. Mech. Appl., 444, 403-414.

[8] Bagarello, F., Di Salvo, R., Gargeno, F., Olivieri, F., 2018. (H,p)induced dynamics and large time behaviours. Physica A: Stat. Mech. Appl., DOI:10.1016/j.physa.2018.03.090. 
[9] Basak, S., 2005. Asset Pricing with Heterogeneous Beliefs. J. Bank. Finance., 29 (11), 2849-2881.

[10] Basieva, I., Pothos, E., Trueblood, J., Khrennikov, A., Busemeyer, J., 2017. Quantum Probability Updating from zero priors (by-passing Cromwell's rule). J. Math. Psychol., 77, 58-69.

[11] Biasis, B., Bossaerts, P., 1998. Asset Prices and Trading Volume in a Beauty Contest. Rev. Econ. Stud., 65 307-340.

[12] Busemeyer J.R., Bruza, P.D., 2012. Quantum models of cognition and decision. Cambridge University Press.

[13] Chen, Z., Epstein, L.G., 2002. Ambiguity, Risk, and Asset Returns in Continuous Time. Econometrica, 70(4), 1403-1443.

[14] Condie, S., Gauguli, J., 2017. The Pricing Effects of Ambiguous Private Information. J. Econ. Theory, 172, 512-557.

[15] Danilov, V.I., Lambert-Mogiliansky, A., Vergopoulos, 2018. V. Dynamic consistency of expected utility under non-classical (quantum) uncertainty. Theory Decis. https://doi.org/10.1007/s11238-018-9659-7.

[16] Epstein, L.G., Schneider, M., 2003. Recursive Multiple Priors. J. Econ. Theory, 113, 1-31.

[17] Epstein, L.G., Schneider, M., 2008. Ambiguity, Information Quality and Asset Pricing. J. Finance, LXII (1),197-228.

[18] Ghirardato, P. F., Macccheroni, M., Marinacci, M., 2004. Differentiating Ambiguity and Ambiguity Attitude. J. Econ. Theory, 118, 133-173.

[19] Giat, Y., Hackman, S.T., Subramanian, A., 2010. Investment under Uncertainty, Heterogeneous beliefs and Agency Conflicts. Rev. Finan. Stud., 23(4), 1360-1404. 
[20] Glosten, L. R., Milgrom, P. R., 1985. Bid, Ask and Transaction prices in a Specialist Market with Heterogeneously Informed Traders. J. Finan. Econ., 14, 71-100.

[21] Grossman, S., 1976. On the Efficiency of Competitive Stock Market where Traders have Diverse Information. J. Finance, XXXI (2), 573-585.

[22] Guidolin, M., Rinaldi, F., 2013. Ambiguity in asset pricing and portfolio choice: a review of the literature. Theor. Decis., 74, 183-217.

[23] Fama, E.F., 1970. Efficient Capital Markets, A Review of Theory and Empirical Work. J. Finance, 25, 383-417.

[24] Harrison, M., Kreps, D., 1978. Speculative Investor Behaviour in a Stock Market with Heterogeneous Expectations. Q. J. Econ., 89, 323-336.

[25] Haven, E., 2008. Private Information and the "Information Function": A Survey of Possible Uses. Theory Dec., 64, 193-228.

[26] Haven, E., Khrennikov, A., 2013. Quantum Social Science, Cambridge University Press.

[27] Haven, E., Khrennikova, P., 2018. A Quantum-Probabilistic paradigm: non-consequential Reasoning and State Dependence in Investment Choice. J. Math. Econ., DOI: 10.1016/j.jmateco.2018.04.003.

[28] Hawkins, R.J., Aoki, M., Frieden, B.R., 2010. Asymmetric information and macroeconomic dynamics. Physica A: Stat. Mech. Appl., 389, 3565-3571 (2010).

[29] Hawkins, R. J., Frieden, B. R., 2012. Asymmetric Information and Quantization in Financial Economics. Int. J. Mathematics and Math. Sciences, 32012. 
[30] Jarrow, R.A., Protter, P., Shimbo, K., 2010. Asset Price Bubbles in Incomplete Markets. Math. Finance, 20(2), 145-185.

[31] Jovanovic, F., Schinckus, C., 2016. Breaking down the barriers between econophysics and financial economics. Int. Rev. Fin. Analysis, 47(10), 256-266.

[32] Kandel, L.E., Pearson, N.D. 1995. Differential Interpretation of Public Signals and Trade in Speculative Markets. J. Polit. Econ., 103 (4), 831-872.

[33] Khrennikov, A., 2015. Quantum Version of Aumann's Approach to Common Knowledge: Sufficient Conditions of Impossibility to Agree on Disagree. J.Math. Econ., 60, 89-104.

[34] Khrennikova, P., 2016. Application of Quantum Master Equation for Long Term Prognosis of Asset Prices. Physica A: Stat. Mech. Appl., 450, 253-263.

[35] Khrennikova, P., 2017. Modeling Behaviour of Decision Makers with the Aid of Algebra of Qubit Creation- Annihilation Operators. J. Math. Psychol., 78(6), 76-85.

[36] Klibanoff, P., Marinacci, M., Mukerje, S., 2005. A Smooth Model of Decision Making under Ambiguity. Econometrica, 73(6), 1849-1892. Check out additionally

[37] Knutson, B., Samanez-Larkin, G.R., Kuhnen, C.M., 2011 Gain and Loss Learning Differentially Contribute to Life Financial Outcomes. PLoS ONE 6(9): e24390. doi:10.1371/journal.pone.0024390.

[38] Kogan, L., Ross., S., Wang, J., 2006. The Price Impact and survival of Irrational Traders. J. Finance, 61, 195-229.

[39] Kolmogorov, A. N., 1933. Grundbegriffe der Wahrscheinlichkeitrechnung, Ergebnisse Der Mathematik; translated as Foundations of 
Probability, New York: Chelsea Publishing Company, (1950).

[40] Kvam, P.D., Pleskac., Yu., S., Busemeyer, J., 2015. Interference effects of choice on confidence: Quantum characteristics of evidence accumulation. PNAS, 112 (34), 10645-10650.

[41] Mantegna, R.M., Stanley, E., 1999 Introduction to Econophysics. Cambridge: Cambridge University Press.

[42] Mehra, R., Prescott, E.C., 1985. The Equity Premium: A Puzzle. J. Monet. Econ., 15, 145-161.

[43] Miao, J., 2014. Introduction to Economic theory of bubbles. J. Math. Econ., 53, 130-136.

[44] Miller, E., 1977. Risk, Uncertainty and Divergence of Opinion. J. Finance, 32(4), 1151-1168.

[45] Morris, S., 1996. Speculative Investor Behaviour and Learning. Q. J. Econ., 111, 1111-1133.

[46] Nezafat, M., Schroder, M., Wang, Q., 2017. Short-Sale constraints, Information Acquisition and Asset Prices. J. Econ. Theory, 172, 273-312.

[47] Pothos, E.M., Busemeyer, J.R. 2009. A Quantum Probability Explanation for Violations of 'rational' Decision Theory. Proc. Roy. Soc. B, 276, 2171-2178.

[48] Pothos, E. M., Busemeyer, J. R., 2013. Can quantum probability provide a new direction for cognitive modeling? Behav. Brain Sci., 36(3), 255-274.

[49] Pulford, B.D., 2009. Is luck on my side? Optimism, pessimism and ambiguity aversion. Q. J. Exp. Psychol., 62(6), 1079-1087. 
[50] Scheinkman, J., Xiong, W., 2003. Overconfidence and Speculative Bubbles. J. Polit. Econ., 111, 1183-1219.

[51] Shiller, R., 2003. Irrational Exuberance, Princeton, Princeton University Press.

[52] Shiller, R., 2014. Speculative Asset Prices. Amer. Econ. Rev., 104(6), 1486-1517.

[53] Tirole, J., 1982. On the Possibility of Speculation under Rational Expectations. Econometrica, 50(5), 1163-1181.

[54] Tversky, A., Kahneman, D., 1973. Availability: A Heruristic for Judging Frequency and Probability. Cogn Psychol., 5, 207-232.

[55] Wang, Z., Solloway, T., Shiffrin, R.M., Busemeyer, J R., 2014, Context effects produced by question orders reveal quantum nature of human judgements. P. Nat. Acad. Sci., 111, 94319436.

[56] Xiong, W. 2013. Bubbles, Crises, and Heterogeneous Beliefs. In J. Fouque J. Langsam (Eds.), Handbook on Systemic Risk (pp. 663-713). Cambridge: Cambridge University Press. doi:10.1017/CBO9781139151184.033 